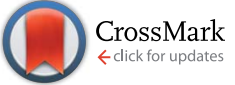

Cite this: RSC Adv., 2016, 6, 9304

Received 6th October 2015

Accepted 4th January 2016

DOI: $10.1039 / \mathrm{c} 5 \mathrm{ra} 20684 \mathrm{~g}$

www.rsc.org/advances

\section{Effect of different fabrication methods on the chemo-physical properties of silk fibroin films and on their interaction with neural cells $\uparrow$}

\author{
Anna Sagnella, $t^{\mathrm{a}}$ Assunta Pistone, $\psi^{\mathrm{b}}$ Simone Bonetti, ${ }^{\mathrm{b}}$ Anna Donnadio, ${ }^{\mathrm{c}}$ \\ Emanuela Saracino, ${ }^{\mathrm{b}}$ Morena Nocchetti, ${ }^{\mathrm{c}}$ Chiara Dionigi, ${ }^{\mathrm{b}}$ Giampiero Ruani, ${ }^{\mathrm{b}}$ \\ Michele Muccini, ${ }^{b}$ Tamara Posati, ${ }^{\star d}$ Valentina Benfenati ${ }^{\star d}$ and Roberto Zamboni ${ }^{d}$
}

In this study, we investigated the influence of processing methods on the chemo-physical properties of silk fibroin (SF) film and on their interaction with neural cells. Structural, thermal and morphological analysis revealed a strong correlation between the conformation, stability and texture of silk films and the fabrication conditions. An increase in temperature, methanol treatment and the use of a microfluidic approach led to an improvement in SF film stability in terms of $\beta$-sheet content, mechanical resistance, dissolution and enzymatic degradation. An effect on the interaction of SF films with neural cells, through a modulation of the surface properties, was also observed. In particular, hydrophobic surfaces induce proliferation of astrocytes and neuron adhesion whereas hydrophilic surfaces promote a remarkable neurite outgrowth. A detailed knowledge of the effect of manufacturing parameters on SF film properties can facilitate and extend the applications of silk-based biomaterials in tissue engineering and drug release systems.

\section{Introduction}

The control of the chemo-physical properties of a biomaterial is an essential and important subject in the biomedical and pharmaceutical fields and in tissue engineering research. Depending on the application site, the specific structure of the material, mechanical properties, wettability and degradability behaviour are all required to ensure that it can fulfil the functions of biomaterials. ${ }^{1-3}$ Silk fibroin (SF) produced by the silkworm Bombyx mori (B. mori) is a natural protein that has been largely employed in the biomedical and tissue engineering fields to make protein-based-biomaterials. ${ }^{4}$ The current advantage of using Bombyx silk is the possibility to obtain, by extraction of fibroin from the cocoon, a pure protein water-solution, known as regenerated silk fibroin, easy to process and to generate a wide variety of silk-based biomaterials such as hydrogels, foams,

${ }^{a}$ Laboratorio di Micro e Submicro Tecnologie abilitanti dell'Emilia-Romagna (MIST ER), Via P. Gobetti 101, I-40129 Bologna, Italy

${ }^{b}$ Consiglio Nazionale delle Ricerche-Istituto per lo Studio dei Materiali Nanostrutturati (CNR-ISMN), Via P. Gobetti 101, 40129 Bologna, Italy

${ }^{c}$ Dipartimento di Scienze Farmaceutiche, Università di Perugia, Via del Liceo 1, 06123, Perugia, Italy

${ }^{d}$ Consiglio Nazionale delle Ricerche - Istituto per la Sintesi Organica e la Fotoreattività (CNR-ISOF), Via P. Gobetti 101, 40129Bologna, Italy.E-mail: tamara.posati@isof.cnr. it; valentina.benfenati@isof.cnr.it

$\dagger$ Electronic supplementary information (ESI) available. See DOI: 10.1039/c5ra20684g

\$ These authors contributed equally. electrospun mats, sponges, films and 3D scaffolds. ${ }^{5}$ Currently, many studies are targeted at the development of a silk platform with specific and controlled features. In particular, tuning the chemo-physical properties of silk formats is possible thanks to the high sensibility of fibroin solution to the following conditions: protein concentration, $\mathrm{pH}$ value, presence of ions, treatment with organic solvents, temperature, shear force and mechanical stress. ${ }^{6-8}$ The type of secondary structure ( $\alpha$-helix, $\beta$ sheet, random-coils) in which the fibroin self-assembles, influences its properties; in fact, it is known that SF can exist in two different conformations: silk I water-soluble (predominance of $\alpha$-helix and random-coil secondary structures) and silk II waterinsoluble ( $\beta$-sheet secondary structures). ${ }^{9}$ The fibroin chains in the aqueous solution are self-assembled mostly in random-coils and $\alpha$-helix structures; by changing only one of the conditions described above, it is possible to induce protein conformation transitions in a controlled manner. Among the different silkbased biomaterials, silk fibroin films have been extensively studied in biomedical applications such as drug delivery ${ }^{\mathbf{1 0}, 11}$ and implantable devices ${ }^{\mathbf{1 2}}$ thanks to their remarkable mechanical properties, biodegradability and their capability to support adhesion, proliferation, and differentiation of different cell types in vitro. ${ }^{\mathbf{1 3 4 1 4}}$ It is known that the surface properties of polymeric interfaces ${ }^{\mathbf{1 5 , 1 6}}$ are important tools to regulate cell shape, polarity (orientation), adhesion, spreading, growth ${ }^{17,18}$ and to regenerate neurites. ${ }^{19}$ Therefore, controlling the surface and chemophysical properties of a biomaterial is fundamental to engineering tissues able to mimic in vivo cell-matrix interactions. ${ }^{20}$ In 
our lab, silk fibroin films were widely characterized as biofunctional interfaces to study neural cells in vitro, like primary cultures of cortical atrocytes ${ }^{21}$ and dorsal root ganglion (DRG) neurons. ${ }^{22}$ In this work, we reported the fabrication and characterization of various SF films using different approaches. First, we studied the effects of temperature, deposition method and methanol treatment on the chemo-physical properties of SF film using several characterization techniques. An effect on the structure, stability, mechanical resistance, morphology and wettability of SF film was observed when changing the film fabrication conditions. We focused on these different methods because they were easy to perform, easy to replicate and already proven for SF film fabrication. Some of these approaches have been thoroughly investigated but, as far as we know, there is no exhaustive investigation that describes in a comparative way their influence on the properties of silk fibroin. Finally, the influence of the different SF surface properties (wettability, morphology) on the biological activity of neural cells was investigated. In particular, comparative studies were carried out proving: (i) the long-term biocompatibility for primary cultures of cortical astrocytes; (ii) support of adhesion and differentiation of primary sensory neurons. We chose these primary cell cultures as they are well validated and characterized models to study the molecular functional properties of astrocytes $^{\mathbf{2 1}}$ as well as the regenerative processes in peripheral neurons. ${ }^{22}$ Overall, the present study allowed us to gain more information on how manufacturing parameters can control and tune the SF film properties facilitating and increasing the applications of silk-based materials in tissue engineering and drug release systems.

\section{Results and discussion}

\section{Preparation of SF films}

Fig. 1 schematizes the different approaches used to prepare SF-based biomaterial films from regenerated SF water- solution: (i) protein solution cast on glass substrates and dried under a hood at room temperature (SF-DC) or dried in an oven at $50{ }^{\circ} \mathrm{C}$ (SF-DO) (Fig. $1 \mathrm{~A}$ and B, respectively); (ii) silk solution deposited on glass substrate by vertical deposition (SF-VD) and dried in an oven at $50{ }^{\circ} \mathrm{C}$ (Fig. 1C); (iii) SF-DC films treated in $\mathrm{MeOH}$ (SF-DC/MeOH) (Fig. 1D). ${ }^{23}$ The vertical deposition can be considered a microfluidic process, since it originates from the flow of the particle suspension into the deposits of particles already formed under the driving force of solvent evaporation. ${ }^{24}$ In vertical deposition, both lateral capillary force (i.e. attractive force between the particles) and surface tension are involved in the movement of particles from the water surface to the deposit on an adequate substrate. Self-assembly of fibroin in water is explained by a model that considers the fibroin molecule like a sequence of hydrophilic-hydrophobic-hydrophilic fragments; this structure sustains the formation of irregularsized micelles depending on chain folding and hydrophobic interactions. ${ }^{25}$ For this reason, the influence of the above mentioned forces on the final fibroin structure cannot be excluded. ${ }^{26}$

\section{Optical transparency of SF films}

Fig. 2A shows the transmission spectra of SF films obtained with the methods described above. All of the films were highly transparent (up to $95 \%$ ) in the visible region (300-800 nm) with a clear consistent decrease below $277 \mathrm{~nm}$ as a result of protein absorbance. The difference in the absorption at $277 \mathrm{~nm}$ for the various samples is due to the different and non-homogeneous thickness of the films that is strictly related to the approaches used for film fabrication. The high transparency of these SF films in the visible range makes them good candidates as optical substrates ${ }^{27}$ and interfaces that allow the optical imaging of cellular activity. ${ }^{21,22,28}$
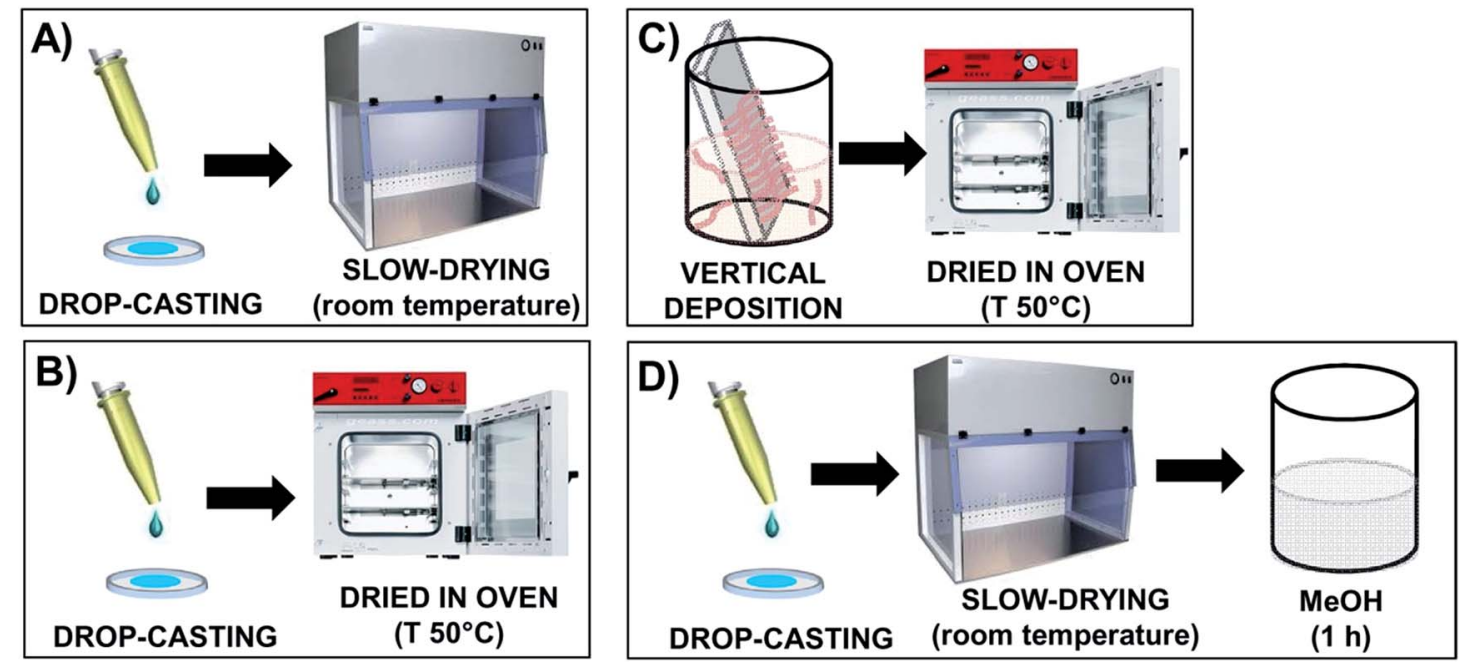

Fig. 1 Scheme of the different approaches used for fabricating SF-based biomaterial films: (A) drop-casting and slow-drying (SF-DC), (B) dropcasting and dried in an oven at $50^{\circ} \mathrm{C}$ (SF-DO), (C) vertical deposition in an oven at $50^{\circ} \mathrm{C}$ (SF-VD), (D) SF-DC films dipped in MeOH for $1 \mathrm{~h}$ (SF-DC/ $\mathrm{MeOH}$ ). 

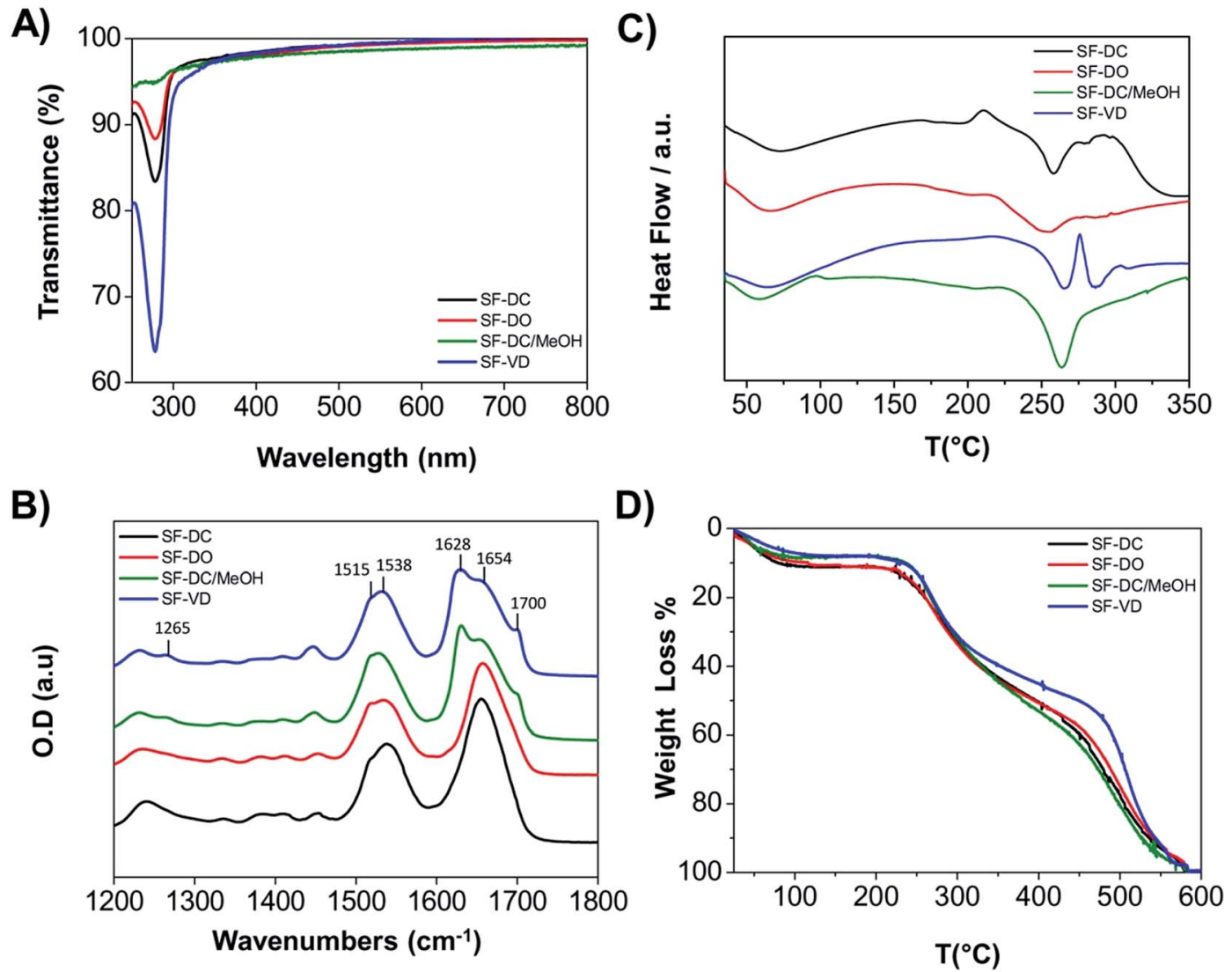

Fig. 2 Optical, structural and thermal properties of different SF films: SF-DC (black lines), SF-DO (red lines), SF-VD (blue lines) and SF-DC/MeOH (green lines). Specifically, (A) optical transparency of silk films recorded in UV-Vis range (300-800 nm); (B) FT-IR vibration absorption spectra collected in amide regions (1200-1800 $\mathrm{cm}^{-1}$ ), thermal profiles obtained by DSC (C) and TGA (D) analysis.

Effect of the different fabrication methods on the secondary structure, thermal behaviour and mechanical properties of SF films

Conformational changes of free-standing SF films were determined by FT-IR analysis. The spectral region from 1700 to 1500 $\mathrm{cm}^{-1}$ was attributed to the absorption by the peptide backbones of amide I (1700-1600 $\left.\mathrm{cm}^{-1}\right)$ and amide II (1600-1500 $\left.\mathrm{cm}^{-1}\right)$, giving information on the different protein secondary structures. ${ }^{29}$ As shown in Fig. 2B, all of the FT-IR spectra showed the $1648-1654 \mathrm{~cm}^{-1}$ and $1535-1542 \mathrm{~cm}^{-1}$ bands, assigned to silk I conformation (random coil) ${ }^{29,30}$ and the $1515 \mathrm{~cm}^{-1}$ band, due to $\mathrm{C}-\mathrm{C}$ stretching of the aromatic ring and $\mathrm{C}-\mathrm{H}$ bending of tyrosine residues in the side chains, ${ }^{30}$ corresponding to silk II conformation. On the other hand, only the FT-IR of SF-VD and SF-DC/MeOH films displayed the typical peaks attributed to silk II conformation at $1700 \mathrm{~cm}^{-1}$ ( $\beta$-sheets intermolecular) and $1628 \mathrm{~cm}^{-1}$ ( $\beta$-sheets intramolecular) in the amide I region; moreover, in the amide III, besides the $\beta$-sheet band, the signal for pure turn structure at $1265 \mathrm{~cm}^{-1}$ was observed. ${ }^{30}$

To better quantify the observed changes in FT-IR spectra, we performed a determination of secondary structure elements using deconvolution of amide I and amide II bands. The results of curve fitting are shown in Fig. S1 and Table S1. $\dagger$ Measurements showed that the SF-VD sample contained the highest amount of $\beta$-sheet structures $(\sim 37 \%)$ compared with SF-DC/
$\mathrm{MeOH}(\sim 18 \%)$, SF-DO $(\sim 4 \%)$ and SF-DC $(\sim 1.5 \%)$ samples. These results indicate that the drop-casting approach for film fabrication followed by solvent evaporation in an oven at $50{ }^{\circ} \mathrm{C}$ does not change the silk protein conformation significantly. Indeed, the infrared spectra for SF-DC and SF-DO films are quite similar with only a slight increase in the band at $1515 \mathrm{~cm}^{-1}$ corresponding to the silk II conformation. On the other hand, at the same temperature, the vertical deposition process (SF-VD film), involving lateral capillary forces and surface tension, leads to evident changes in the self-assembly of polypeptide chains greater than those observed for SF-DC/MeOH films. The presence of silk II conformation in SF films treated with $\mathrm{MeOH}$ is in agreement with literature data. ${ }^{23}$ In Fig. 2C, DSC curves for SFDC, SF-DO, SF-VD and SF-DC/MeOH films are reported. All samples showed an endothermic peak between 50 and $100{ }^{\circ} \mathrm{C}$ that can be assigned to the loss of adsorbed weakly bonded water. The SF-DC sample showed a non-isothermal crystallization peak at around $213{ }^{\circ} \mathrm{C}$ due to the transition of unstable noncrystalline structures to $\beta$-sheets in agreement with data reported by Kaplan and colleagues. ${ }^{29,30}$ Above this crystallization temperature, the film started to degrade, with an endothermic peak at around $257{ }^{\circ} \mathrm{C}$. A less pronounced crystallization peak was observed in the SF-DO DSC curve indicating partial protein crystallization to $\beta$-sheets. The degradation peak appears broader than in the SF-DC film, due to the probable coexistence of the 
protein in silk I and $\beta$-sheet conformations having different degradation temperatures. After treatment with methanol, the film SF-DC/MeOH crystallized to form $\beta$-sheets (see green line in Fig. 2B). The endothermic peak $\left(\sim 50{ }^{\circ} \mathrm{C}\right)$ related to the loss of adsorbed water decreased in intensity and shifted to lower temperature, implying that methanol-treated silk film absorbed a lower content of water and that the silk-water interaction was weaker. As expected, the crystallization peak was absent because the protein was in $\beta$-sheet conformation; on the other hand, the degradation peak increased to about $264{ }^{\circ} \mathrm{C}$, suggesting a higher thermal stability in comparison to SF-DC and SF-DO samples. For the SF-VD sample, a similar thermal behavior was observed until $250{ }^{\circ} \mathrm{C}$. Above this temperature, a significant difference was detected: two degradation peaks appeared at around $266{ }^{\circ} \mathrm{C}$ and $287^{\circ} \mathrm{C}$. Both were higher than the previous ones and this result could be related to the stability induced in the samples by the $\beta$ sheet structures. Since the stability of the protein is related to its degree of crystallinity, the methanol treatment, and especially the vertical deposition method, induced the formation of a more stable crystalline structure of silk, according to the FT-IR results.

Finally, TGA data for the different SF samples, conditioned at a relative humidity of $75 \%$, confirmed weaker water-silk interactions following the increase in silk II content (Fig. 2D). In fact, the water content decreased by $11.2 \%, 10.6 \%, 8.6 \%$ and $7.7 \%$ for SF-DC, SF-DO, SF-DC/MeOH, and SF-VD samples, respectively; SF-DC and SF-DO samples, characterized by a similar secondary structure (random coil and $\alpha$-helix, see Fig. 2B) showed a comparable weight loss, whereas the SF-DC/MeOH and SF-VD samples displayed a decreased amount of water because of the predominance of $\beta$-sheet structures. These data are in agreement with those reported in the literature. ${ }^{\mathbf{9 9 , 3 0}}$

The flexibility and robustness of silk films were analyzed by stress-strain tests. As reported in Table 1, the value of the tensile modulus of different SF films increased as a consequence of an increase in $\beta$-sheet content, indicating a mechanical stability due to the higher amount of physical cross-links between fibroin chains. In this respect, the SF-VD sample with the highest amount of $\beta$-structures $(\sim 37 \%)$ showed the highest tensile modulus $E$. These results show that the mechanical properties of silk films can be controlled by varying the different processing approaches.

\section{Morphological characterization of the SF films}

The morphology of the different SF films was investigated by optical microscopy and AFM analysis. Micrographs obtained by

Table 1 Mechanical parameters (tensile modulus $E$, stress at breaking (UTS) and elongation at breaking $(\varepsilon)$ ) of the SF films prepared by different processes

\begin{tabular}{llll}
\hline & $E / \mathrm{N} \mathrm{mm}^{-2}$ & $\mathrm{UTS} / \mathrm{N} \mathrm{mm}^{-2}$ & $\varepsilon / \%$ \\
\hline SF-DC & $2575 \pm 23$ & $76.5 \pm 0.4$ & $3.39 \pm 0.47$ \\
SF-DO & $2562 \pm 96$ & $68.8 \pm 7.2$ & $2.96 \pm 0.59$ \\
SF-DC/MeOH & $3262 \pm 170$ & $68.6 \pm 2.1$ & $2.16 \pm 0.07$ \\
SF-VD & $3815 \pm 104$ & $67.1 \pm 6.0$ & $2.82 \pm 0.21$
\end{tabular}

optical microscopy showed a different texture of the SF films (Fig. S2 $\dagger$ ). SF-DC and SF-DO samples appeared quite homogeneous, uniform, and very similar (Fig. S2A and $\mathrm{C}^{\dagger}$ ). On the other hand, the SF-VD film (Fig. S2B $\dagger$ ) displayed a non-homogeneous texture with the presence of "valleys and ridges" related to the intrinsic characteristic of the vertical deposition method. ${ }^{24,31}$ Finally, the SF-DC/MeOH (Fig. S2D $\dagger$ ) sample appeared cracked probably as a result of the strong and rapid dehydration process induced by the methanol treatment. Atomic force microscopy (AFM) is a powerful tool to obtain direct information about the surface topography of a film with a nanometer spatial resolution. AFM images of the various SF films (Fig. 3) were collected to detect the morphological differences induced by the different approaches used for film fabrication. The SF-DC sample with a secondary structure of random coil type showed the smoothest surface with an average roughness $\left(R_{\mathrm{a}}\right)$ of about $6 \mathrm{~nm}$ (Fig. 3A and Table 2). The light-grainy morphology of this film is similar to that observed for other SF films obtained using the same drop-casting and slow-drying method. ${ }^{32}$ A similar texture but with an increased $R_{\mathrm{a}}(\sim 51 \mathrm{~nm})$ was observed for SF-VD film (Fig. 3C and Table 2). On the other hand, films treated with methanol (SF-DC/MeOH) and dried in an oven (SF-DO) showed a completely different morphology (Fig. 3B and D, respectively) with intermediate $R_{\mathrm{a}}$ values ( $\sim 29 \mathrm{~nm}$ and $\sim 15 \mathrm{~nm}$, respectively, see Table 2). In particular, the SF-DO film was characterized by little holes and by small aggregates of protein of around 500 $\mathrm{nm}$; in this case, the drying temperature did not affect the conformational structure of the protein that was similar to that of the SF-DC sample (silk I conformation) but influenced the morphology of the SF film surface. Finally, the SF-DC/MeOH sample showed a morphology totally different from that of the SF-VD film with the same secondary structure ( $\beta$-sheet type); indeed, the surface presented the highest degree of molecular aggregation (aggregates of proteins of around $1.5 \mu \mathrm{m}$ ) suggesting that methanol treatment could promote a more packed protein structure (increase in crystallinity index). ${ }^{32}$ The different
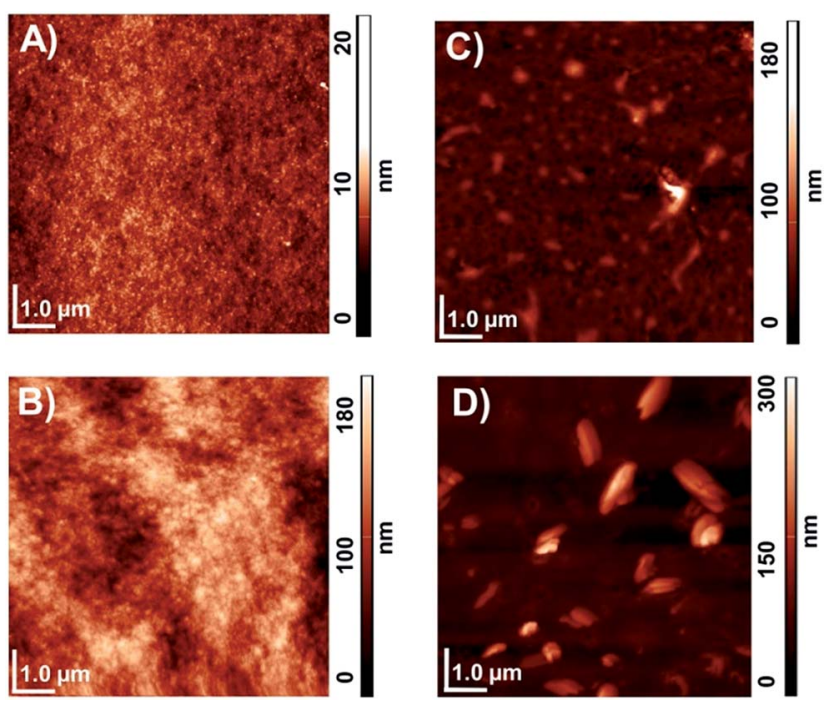

Fig. 3 AFM topographical images of SF films obtained using different processes: (A) SF-DC, (B) SF-VD, (C) SF-DO and (D) SF-DC/MeOH 
Table 2 Contact angle (CA) and average roughness $\left(R_{\mathrm{a}}\right)$ values of the SF films prepared by different processes

\begin{tabular}{lclll}
\hline & SF-DC & SF-DO & SF-DC/MeOH & SF-VD \\
\hline $\left.\mathrm{CA}{ }^{\circ}{ }^{\circ}\right)$ & $39.1 \pm 0.45$ & $45.9 \pm 0.30$ & $53.9 \pm 0.25$ & $65.8 \pm 0.80$ \\
$R_{\mathrm{a}}{ }^{3}$ & $6.0 \pm 1.2$ & $15.5 \pm 3.4$ & $29.1 \pm 5.5$ & $51.2 \pm 7.5$
\end{tabular}

${ }^{a}$ Measured by AFM.

morphologies and features of the four films analyzed are probably due to the different molecular motion of the protein induced by the different conditions of water evaporation used for film preparation.

\section{Effect of the fabrication methods on the enzymatic degradation and dissolution of SF films}

Several studies have demonstrated that the degree of degradation of various silk-based biomaterials could be controlled by the content of silk II structures. ${ }^{33}$ For this reason, we tested the resistance to enzymatic degradation (protease XIV solution) and the rate of dissolution in aqueous DMEM (Dulbecco's Modified Eagle Medium) of fibroin assembled in the different films. The biodegradation experiment was performed by incubating the different SF films at $37{ }^{\circ} \mathrm{C}$ with protease XIV solution for 15 days. The rate of degradation was calculated through the weight loss of fibroin in the films. The results, reported in Fig. 4A, confirmed a correlation between the content of silk I $(\alpha$-helix and random coil) and silk II structures ( $\beta$-sheets) and the rate of degradation. Indeed, SF-DC and SF-DO films (Fig. 4A, black and red line, respectively) characterized by the predominance of silk I water-soluble structure (only $1.5 \%$ and $4 \%$ of $\beta$-sheet structures, respectively) lost $\sim 90 \%$ of their initial weight after $24 \mathrm{~h}$ of incubation. On the other hand, the SF-VD and SF-DC/MeOH films (Fig. 4A, blue and green line, respectively), characterized by silk II water-insoluble conformation, showed a lower rate of degradation compared to SF-DC and SF-DO samples. In particular, SF-DC/MeOH film displayed a weight loss of $\sim 70 \%$, $\sim 85 \%$ and $\sim 90 \%$ after $24 \mathrm{~h}, 48 \mathrm{~h}$ and 1 week of incubation, respectively, whereas the SF-VD film, characterized by the highest content of $\beta$-sheet structures $(\sim 37 \%$ instead of $\sim 18 \%$ of the SF-DC/MeOH sample) showed the lowest rate of degradation losing $\sim 20 \%$ after $48 \mathrm{~h}, \sim 30 \%$ after 1 week and $\sim 70 \%$ after 15 days.

Fig. 4B shows the dissolution profiles for the different prepared SF films. SF-DC (black line) and SF-VD (blue line) samples revealed the highest and lowest rates of dissolution in DMEM medium, respectively. Indeed, after $6 \mathrm{~h}$ of incubation in DMEM, the dissolved percentage of fibroin films was $~ 95 \%$ for SF-DC and $<10 \%$ for SF-VD; moreover, for the SF-VD sample, the maximum weight loss was $\sim 20 \%$ after 15 days. Regarding the SF-DO and SF-DC/MeOH films (Fig. 4B, red and green line, respectively), they showed intermediate dissolution profiles in DMEM medium; SF-DO film lost $~ 70 \%$ of weight after $6 \mathrm{~h}$ then a plateau was observed, while SF-DC/MeOH film displayed a lower dissolution rate losing $\sim 50 \%$ of weight after $6 \mathrm{~h}$ and
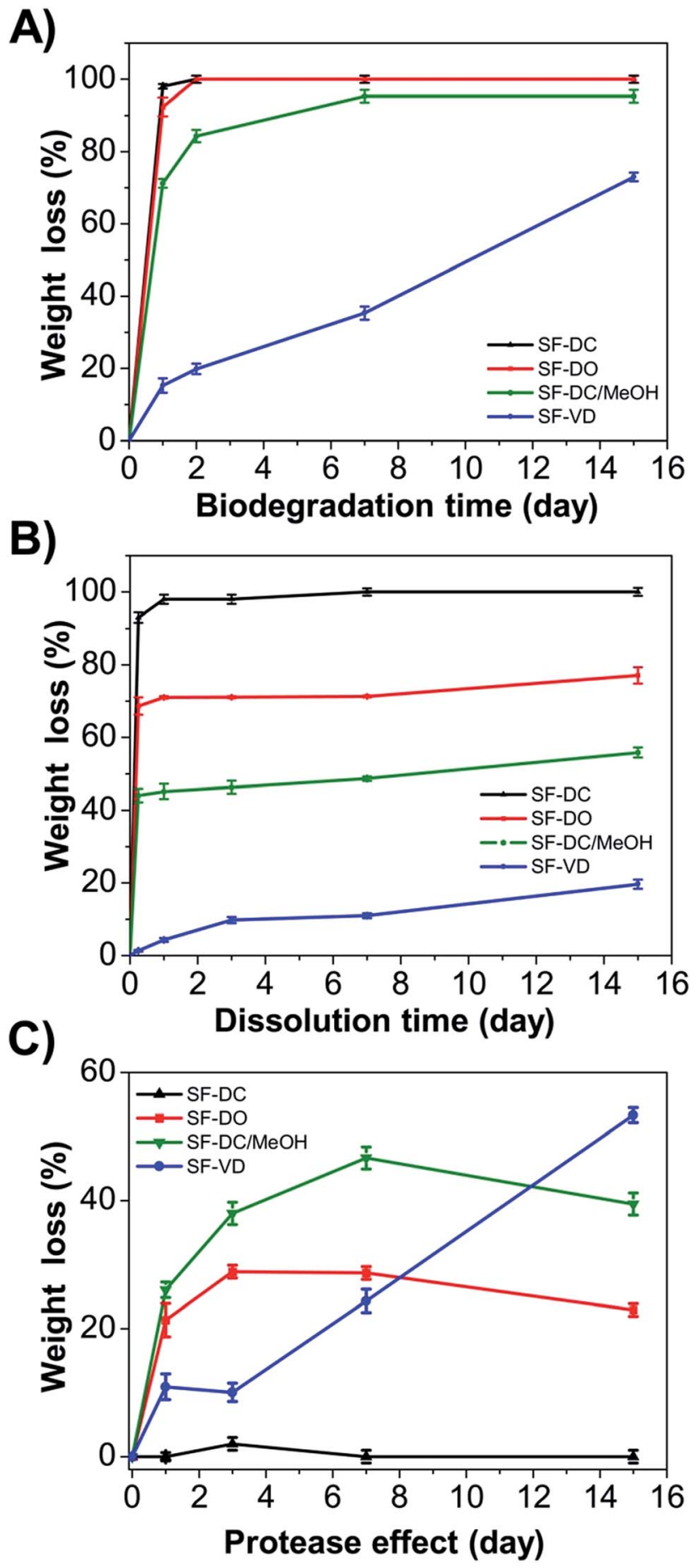

Fig. 4 Biodegradation and dissolution profiles for SF films: SF-DC (black line), SF-DO (red line), SF-VD (blue line) and SF-DC/MeOH (green line). Specifically, (A) biodegradation rate of silk films incubated in protease XIV solution at $37^{\circ} \mathrm{C}$; (B) dissolution time of silk films maintained in aqueous DMEM medium at $37^{\circ} \mathrm{C}$; (C) enzymatic effect profiles on different silk films.

$\sim 55 \%$ after 15 days of incubation. Dissolution profiles, according to biodegradation data, highlighted the fact that the fibroin conformations affected the solubility of protein assembled in various films. In particular, with an increase in silk II 
structures, the amount of dissolved silk fibroin per unit time decreased. The different behaviour of the SF-VD and SF-DC/ $\mathrm{MeOH}$ films in DMEM and in the presence of protease could be due to the more stable crystalline structure of SF-VD (see DSC data, Fig. 2C and deconvolution of amide I and amide II bands, Fig. S1 and Table S1 $\dagger$ ) and to the different morphologies of silk films after the different preparations and treatments.

Indeed, the cracks generated by the methanol treatment (see optical images in Fig. S2D†) can give rise to breaking points on the films increasing the exposed surface of silk to the proteolytic enzyme. Notably, by subtracting the weight loss values correlated to the dissolution profiles (Fig. 4B) from those of the biodegradation assay (Fig. 4A), we calculated the effective enzymatic degradation of silk films (Fig. 4C). The results displayed an increase in protease action corresponding to a decrease in dissolution rate of silk films and to an increased content of silk II structure. Indeed, the enzymatic degradation effect was almost zero for the water-soluble SF-DC sample, characterized by the lowest content of $\beta$-structures $(\sim 1.5 \%)$ (black line) and of about $60 \%$ after 15 days for water-insoluble SF-VD (blue line) films with a $\beta$-sheet content of $\sim 37 \%$. An intermediate protease effect for SF-DO and SF-DC/MeOH films (red and green line, respectively) was observed according to their $\beta$-sheet content ( $\sim 4$ and $\sim 18 \%$, respectively).

\section{Surface energy of the different prepared SF films and their interaction with neural cells}

Among the surface properties of a material, the wettability is the one that mainly affects cell adhesion. The degree of wetting is determined by a balance between adhesive and cohesive forces, determined by the hydrophobicity/hydrophilicity of the surface. ${ }^{34}$ Recent studies demonstrated that surface modifications of SF films were used to permit controlled adsorption of proteins and regulated cell proliferation. ${ }^{32}$ Traditionally, values of water contact angles between $50^{\circ}$ and $70^{\circ}$ are reported for SF film depending on the post-treatment of the films. ${ }^{35}$ In this work, we performed DMEM medium contact angle measurements to investigate the surface properties of the SF films prepared using different methods (Table 2). The SF-DC film (Fig. S3A $\dagger$ ) showed the lowest value of contact angle $\left(\sim 39.1^{\circ}\right.$, see Table 2) attributed to the significant hydrophilicity/wettability of the surface. The increase in contact angle values for SF-DO, SF-DC/MeOH and SF-VD films $\left(\sim 45.9^{\circ}, \sim 53.9^{\circ}\right.$ and $\sim 65.8^{\circ}$, respectively, see Table 2 ) indicated minor wettability of the surfaces related to increased hydrophobicity (Fig. S3B-D $\dagger$ ). Notably, the wetting of the SF films decreased with the increase in $\beta$-sheet content (see Fig. 2). This result is in agreement with the different dissolution and degradation profiles observed for the SF substrates (see Fig. 4B). In fact, the most hydrophobic SFVD film is the one that dissolves and degrades most slowly unlike the most hydrophilic SF-DC film that shows the highest degree of degradation and dissolution.

It is well established that morphology and surface properties of substrates influence cell behaviour in terms of adhesion, proliferation and morphology. ${ }^{36}$ In particular, biological activity of silk fibroin substrates has been extensively investigated. ${ }^{34}$ To test how surface features and fabrication methods affect the behaviour of SF films as neural interfaces, primary cultures of rat cortical astrocytes were plated on the different SF substrates. The cultures were maintained for 4 weeks in vitro and adhesion, morphology and viability were investigated at different time points.

As shown in Fig. 5A-D, after 1 day in vitro (DIV), adherent spherical astrocytes appeared on SF-DC and SF-VD substrates (Fig. 5A and D, respectively), whereas SF-DO and SF-DC/MeOH films (Fig. 5B and $\mathrm{C}$, respectively) induced spreading of the cell bodies on the substrate surface. After 28 DIV, by staining cells with fluorescein diacetate (FDA) (Fig. 5E-H), astrocytes appeared to be still alive on all the SF substrates but showed different morphologies. In particular, confluent astrocytes were visible on SF-DC, SF-DO and SF-DC/MeOH substrates (Fig. 5E$\mathrm{G})$. However, astrocytes only showed the typical polygonal shape on SF-DC and SF-DO films, as already observed for previously reported silk films, ${ }^{21}$ while on the SF-DC/MeOH sample, cells appeared very close and smaller. In contrast, on SF-VD substrates, polygonal astrocytes grew in large islands, alternating with non-adherence areas (Fig. $5 \mathrm{H}$ ). The latter behaviour is probably due to the specific texture, based on "ridges and valleys", that characterizes the surface of SF-VD film (see optical micrograph in Fig. S2B $\dagger$ ). These observations were perfectly in line with the quantitative analysis of long-term cell viability, obtained from the AlamarBlue (AB) assay. As shown in viability curves (Fig. 5I), cell adhesion (after 1 DIV) is roughly comparable for all of the SF films. In contrast, the proliferation of astrocytes was significantly higher for SF-DC/MeOH films indicating that different cell morphologies strongly correlated with the percentage of confluency reached by astroglial cells on different substrates.

Recent studies have shown that cell growth is higher on weakly hydrophilic surfaces (with water contact angles of approximately $60^{\circ}$ ), due to an increased adsorption of ECM proteins. ${ }^{37,38}$ Hence, the increase in $\beta$-sheet content, accompanied by increased hydrophobicity, might be responsible for the different effects of the SF-DC/MeOH substrate on the growth profile and morphology of cultured astrocytes. ${ }^{34}$ The SF-VD film, similar in terms of secondary structure and wettability to the SFDC/MeOH sample, deviates from this trend probably because of the presence of non-adherence areas. A similar effect was also observed in the interaction of SF substrates with dorsal root ganglion (DRG) cultures. Immunofluorescence of neuronal cells with an antibody against neuronal nuclear protein (NeuN), a typical marker expressed by mature neurons, ${ }^{39}$ revealed a different spatial distribution of neuronal nuclei. In particular, spread neurons appeared on SF-DC and SF-DO films (Fig. 6A and B) while clustered DRG neurons adhered on SF-VD and SF$\mathrm{DC} / \mathrm{MeOH}$ substrates (Fig. 6C and D). For quantitative analysis (Fig. 6E), NeuN positive cells were counted and results from a histogram plot suggested a maximal adhesion on the hydrophobic SF-DC/MeOH substrates compared to the other substrates. Of note, the average number of neurons counted on the SF-DC/MeOH substrate was significantly higher compared to SF-DC and SF-DO $(p<0.001)$, while this highly statistically significant difference disappeared with the SF-DV substrate. 


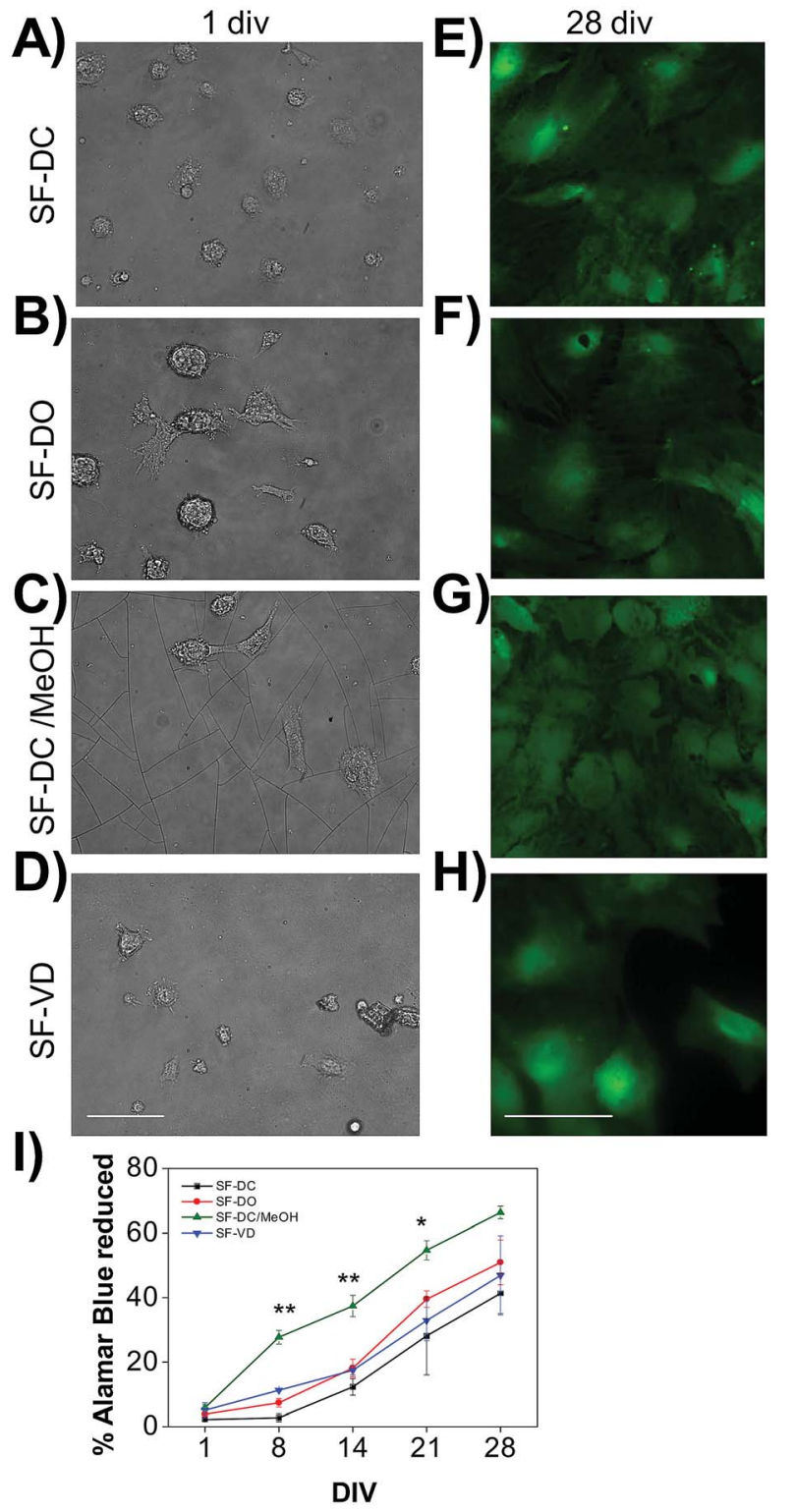

Fig. 5 Cell adhesion and viability on silk substrates. (A-D) Micrographs of astrocytes cultured on (A) SF-DC, (B) SF-DO, (C) SF-DC/MeOH, (D) SF-VD films after 1 DIV: cells are spread on SF-DO and SF-DC/MeOH substrates. $(\mathrm{E}-\mathrm{H})$ Images of FDA-stained astrocytes plated on the same substrates and captured after 28 days in vitro: cells appeared smaller and tight on SF-DC/MeOH film, while polygonal shapes and non-adherence areas are visible on SF-VD films. Scale bar is $100 \mu \mathrm{m}$. (I) Time course of astrocyte viability on different SF substrates, investigated by $A B$ assay at different time points. The data indicated that proliferation of astrocytes was higher on SF-DC/MeOH films. Data are plotted as the average percentages \pm Standard Error (SE) of reduced $\mathrm{AB}$ versus DIV $(* * p<0.01 ; * p<0.05)$.

Also in this case, the amount of $\beta$-sheets coupled with the reduced wettability could promote cluster formation on SF-DC/ $\mathrm{MeOH}$ and SF-VD substrates affecting neuron adhesion.

During cell culture preparation, the neurites of DRG neurons were cut. Once plated, they were able to differentiate regenerating axons in vitro if their health conditions were preserved. Since the surface properties of polymeric interfaces play an
A)

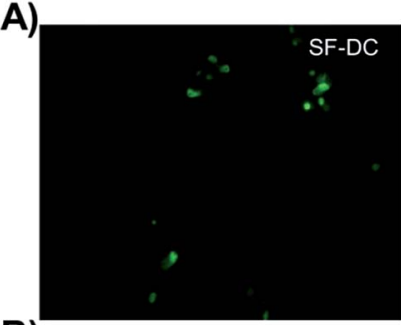

B)
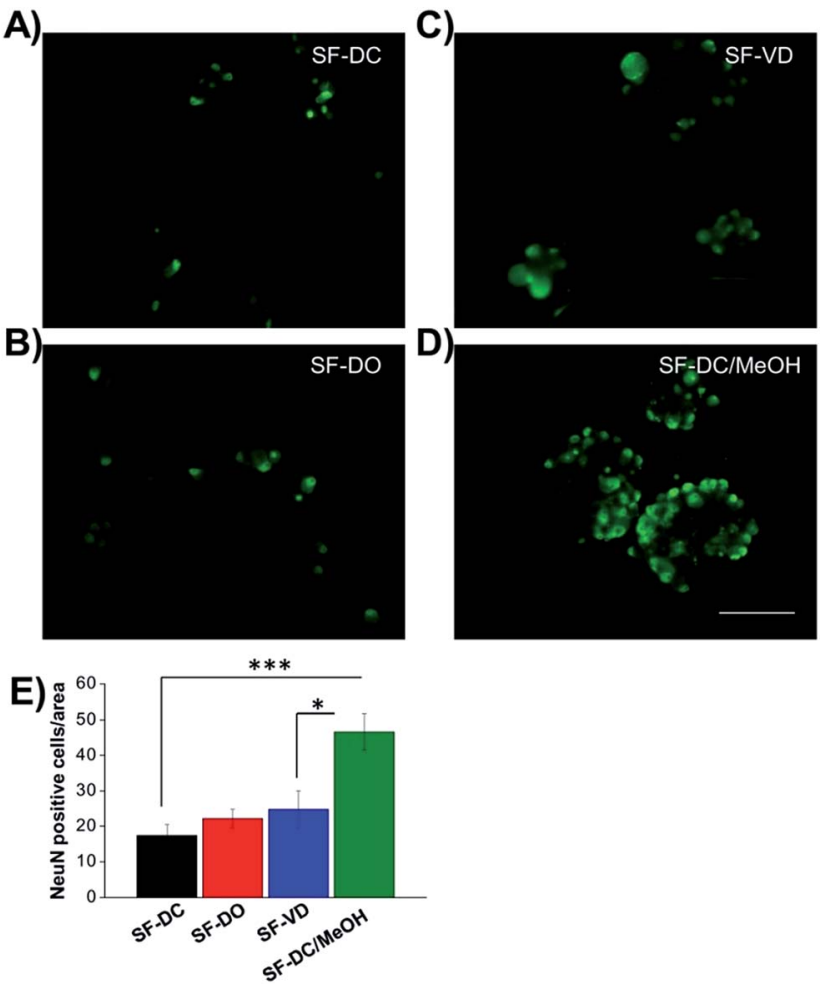

Fig. 6 Neuron adhesion. $20 \times$ magnification of DRG neurons plated on (A) SF-DC, (B) SF-DO, (C) SF-VD, (D) SF-DC/MeOH films stained for the neuronal marker NeuN after 3 DIV. Scale bar is $100 \mu \mathrm{m}$. (E) Histogram plot of the number of NeuN-positive cells/area. The neurons counted on SF-DC/MeOH (green bar) were significantly higher than on the other substrates $(* * * p<0.001, * p<0.05)$.

important role in regenerating nervous damage, ${ }^{19}$ axon regeneration was investigated. Fig. 7A-D reports the micrographs of DRG neuron cultures performed on (A) SF-DC, (B) SF-DO, (C) SFVD, and (D) SF-DC/MeOH films and grown 11 DIV. Morphological observations of neural cells revealed neuron cell bodies with different diameters and process extensions and networking among them in all of the samples, indicating occurrence of axonal regeneration. Networking and neurite length were less evident on SF-DC/MeOH film when compared to the other conditions. Finally, we quantified and compared neurite length after 3 DIV, 9 DIV and 11 DIV (Fig. 7E) under all four experimental conditions. The average neurite length showed a progressive neurite growth over time on substrates SFDC, SF-DO and SF-VD. Notably, after 11 DIV, the average neurite length was significantly higher $(* * *=p<0.001)$ in SF-DO with respect to the other substrates. In contrast, SF-DC/MeOH films showed no neurite outgrowth over time. Notably, in contrast to cell adhesion, neurite outgrowth is promoted on interfaces with increased wettability/hydrophilicity (see Fig. S3†). These data are in agreement with studies showing that PC-12 cells displayed an increased outgrowth of neurites on hydrophilic surfaces rather than on hydrophobic ones. ${ }^{19}$ The difference between the hydrophilic samples (SF-DC and SF-DO) could be a consequence of their different morphologies; probably neurite outgrowth is not favored by highly wettable surfaces alone but also by the presence of an irregular and rough texture. 


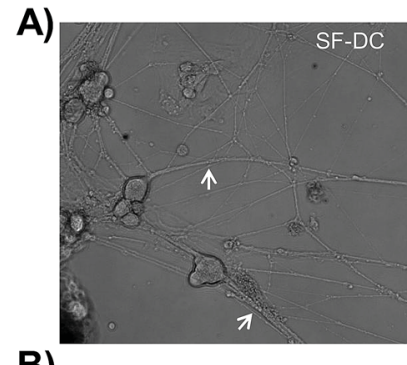

B)
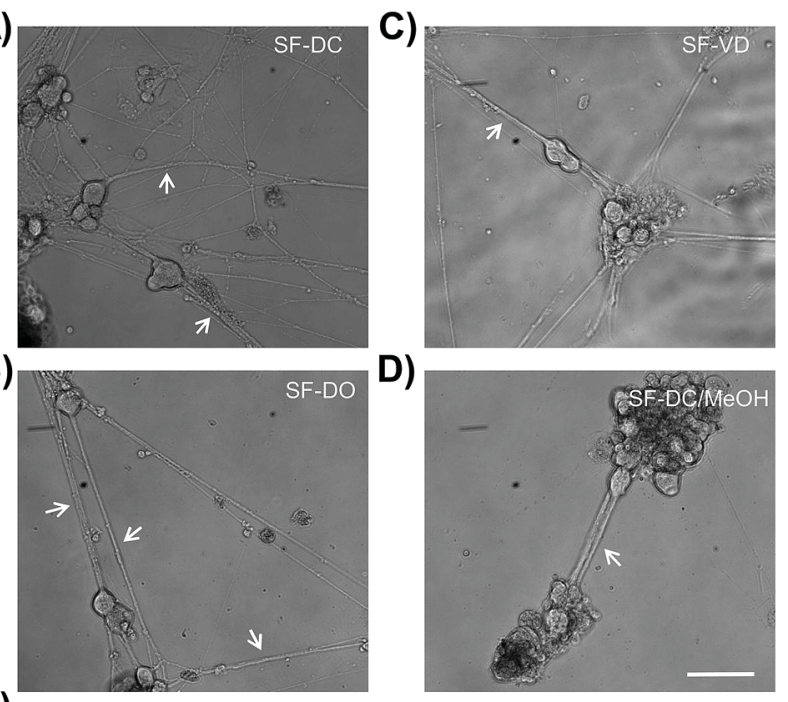

E)
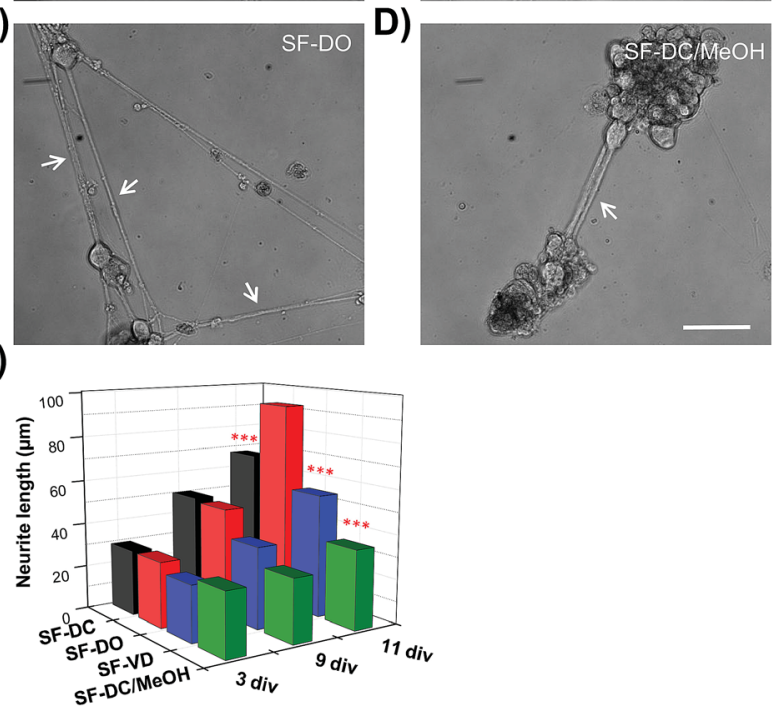

Fig. 7 Neurite outgrowth analysis. Micrographs of DRG neuron cultures performed on (A) SF-DC, (B) SF-DO, (C) SF-VD, and (D) SF$\mathrm{DC} / \mathrm{MeOH}$ films, after 11 days in vitro. Arrowheads indicate neurite outgrowth. Scale bar is $100 \mu \mathrm{m}$. (E) Histogram plot of average neurite length measured in neurons after 3 DIV, 9 DIV and 11 DIV. The neurite length was significantly higher after 11 DIV $(* * * p<0.001)$ for DRG neurons plated on the SF-DO film compared to the other substrates. After 11 DIV, SF-DC average: $67.5 \pm$ SE 3.4; SF-DO average: $93.2 \pm$ SE 6.7; SF-VD average: $55.5 \pm \mathrm{SE}$ 4.9; SF-DC/MeOH average: $35.7 \pm$ SE 7.4 .

\section{Experimental}

\section{Extraction of regenerated silk fibroin}

Regenerated SF water-solution was extracted from B. mori cocoons (CNR-IBIMET, Bologna, Italy). The cocoons were degummed in a boiling $0.02 \mathrm{M} \mathrm{Na}_{2} \mathrm{CO}_{3}$ (Sigma-Aldrich, St Louis, MO, USA) solution for $45 \mathrm{~min}$ to obtain SF fibres that were then rinsed three times in Milli-Q water and dissolved in a 9.3 M LiBr solution at $60{ }^{\circ} \mathrm{C}(4-6 \mathrm{~h})$. The SF solution was subsequently dialyzed (dialysis membranes, MWCO 3500) against distilled water for $48 \mathrm{~h}$ and centrifuged to obtain the pure regenerated SF solutions ( $c a .6-8 \mathrm{w} / \mathrm{v} \%)$. The SF concentration was measured with the gravimetric method by weighing five different films. The SF water-solution was stored at $4{ }^{\circ} \mathrm{C}$.

\section{Fabrication of SF-based biomaterial films}

SF films were fabricated by different methods (Fig. 1).

(1) Drop-casting and slow-drying (SF-DC): an exact volume of silk solution was cast on a glass coverslip and/or polydimethylsiloxane (PDMS) support and then dried under a sterile hood at room temperature (Fig. 1A).

(2) Drop-casting and dried in an oven (SF-DO): an exact volume of SF water-solution was cast on a glass coverslip or PDMS support and then dried in an oven at $50{ }^{\circ} \mathrm{C}$ until the film was completely dry (Fig. 1B).

(3) Vertical deposition (SF-VD): SF water-solution was deposited on a glass coverslip substrate by vertical deposition in an oven at $50{ }^{\circ} \mathrm{C}$ until the film was completely dry (Fig. 1C).

(4) $\mathrm{MeOH}$ treatment (SF-DC/MeOH): films obtained by the drop casting and slow drying approach were immersed in methanol for $1 \mathrm{~h}$ (Fig. 1D). After preparation, the SF-DC/MeOH films were thoroughly washed with water.

To obtain free-standing SF films, SF solutions were dropped on PDMS support, left to dry then peeled off from the substrate.

The round glass coverslip substrates used to deposit SF films (Marienfeld Laboratory Glassware, Microscope cover Glass) were first cleaned by immersion in 100\% ethanol overnight and then dried under an argon flux.

The thickness of all SF films, determined by micrometer, was in the range of $35-40 \mu \mathrm{m}$.

\section{Chemo-physical investigations}

Fourier transform infrared (FT-IR) spectroscopy. The mid IR (400-4000 $\mathrm{cm}^{-1}$ ) absorption measurements were carried out in a Bruker IFS-88 FT-IR interferometer at $4 \mathrm{~cm}^{-1}$ resolution averaging over 512 scans to improve the signal-to-noise ratio. Absorption spectra have been measured on free-standing SF films. The curve-fitting of overlapping bands of the infrared spectra covering the amide I and II regions (1500-1700 $\mathrm{cm}^{-1}$ ) was performed using the Levenberg-Marquardt algorithm implemented in OPUS 2.0 software. Quantitative analysis of the amide I and amide II bands was done using curve fitting, second derivative and Fourier self-deconvolution methods as already reported by $\mathrm{Hu}$ et al. ${ }^{29}$ Spectra were processed using Bruker OPUS software. The amount of $\beta$-sheet component in different samples was computed as the ratio between the sum of the areas of the corresponding peaks in the amide I and amide II bands and the sum of the areas of all of the peaks in the same spectral region.

UV-Vis optical analysis. A Jasco V-550 UV-Vis spectrophotometer was used to obtain the percentage transmittance from the SF films prepared on a quartz substrate.

Thermal analysis. The thermal properties of the silk films were measured in a DSC (Differential Scanning Calorimetry) Instrument (METTLER TOLEDO) under a dry nitrogen gas flow of $70 \mathrm{~mL} \mathrm{~min}^{-1}$. The samples were heated at $2{ }^{\circ} \mathrm{C} \mathrm{min}^{-1}$ from 35 ${ }^{\circ} \mathrm{C}$ to $350{ }^{\circ} \mathrm{C}$. Thermogravimetric analyses (TGA) on different silk films, previously conditioned at $75{ }^{\circ} \mathrm{C}$ of relative humidity (RH) using a saturated solution of $\mathrm{NaCl}$, were performed in air up to $700{ }^{\circ} \mathrm{C}$ (temperature ramp of $2{ }^{\circ} \mathrm{C} \mathrm{min}^{-1}$ ) by a STA1500 system equipped with a simultaneous thermal analyzer.

Silk film degradation and dissolution assay. For the degradation test, free-standing SF films were incubated at $37{ }^{\circ} \mathrm{C}$ in a $3 \mathrm{~mL}$ solution of $1 \mathrm{mg} \mathrm{mL}{ }^{-1}$ protease XIV (protease from Streptomyces griseus type XIV, $\geq 3.5$ units per $\mathrm{mg}$ solid, Sigma) in 
phosphate-buffered saline (PBS) at $\mathrm{pH}$ 7.4. Each solution contained an approximately equivalent mass (50 $\pm 2 \mathrm{mg})$ of silk film (thickness $\sim 40 \mu \mathrm{m}$ ). Solutions were replenished with enzyme and collected daily. At designated time points, groups of samples were rinsed in distilled water and prepared for mass balance. Samples were dehydrated in an oven at $50{ }^{\circ} \mathrm{C}$ for $2 \mathrm{~h}$. Following removal from the oven, the samples were weighed and returned to a new solution with fresh enzyme. Percent weight loss over time was determined. Each experiment was performed in triplicate. For dissolution experiments, SF films were incubated at $37^{\circ} \mathrm{C}$ in a $3 \mathrm{~mL}$ solution of DMEM (Dulbecco's Modified Eagle Medium) at $\mathrm{pH}$ 7.4. The next steps follow the degradation procedure reported above.

Atomic force microscopy (AFM). AFM topographical images were collected using an NT-MDT solver scanning probe microscope in tapping mode with a sample kept in air. The scanning was performed at 50-60 nm oscillation amplitude setting (65\% of the free amplitude at $f_{0}$ ). Sampling was set at $512 \times$ 512 pixels. The standard deviation of the average roughness has been calculated using two different $10 \times 10 \mu \mathrm{m}$ scans of three samples for each condition.

Contact angle measurement (CA). The wettability of the SF films (formed on glass substrate) was investigated by measuring the DMEM contact angles on the SF samples by the static sessile drop method and using a Digidrop GBX Model DS. For each film, at least four drops were measured.

Mechanical tests. Stress-strain mechanical tests were carried out under ambient conditions using a Zwick Roell Z1.0 testing machine with a $200 \mathrm{~N}$ static load cell. The Young's modulus (the slope of the stress-strain curve in the elastic deformation region), the stress at breaking (the tensile stress at the breaking point of the specimen, UTS), and the elongation at breaking (the percentage increase in length that occurs before the sample breaks, $\varepsilon$ ) were measured on rectangular-shaped film strips, obtained using a cutting machine; the length and width of the strips were 50 and $5 \mathrm{~mm}$, respectively. The thickness of the film strip, determined by micrometer with an uncertainty of $1 \mu \mathrm{m}$, was in the range of 35-40 $\mu \mathrm{m}$. An initial grip separation of $10.000 \pm 0.002 \mathrm{~mm}$ and a crosshead speed of $30 \mathrm{~mm} \mathrm{~min}{ }^{-1}$ were used. At least four replicate film strips were analyzed. The data were interpreted with TestXpert V11.0 Master software.

Cell culture preparation. Experiments carried out with animals were performed in concordance to the European Council Law for the protection of laboratory animals, with the approval of the bioethical committee of the University of Bologna. Every effort was made to minimize the number of animals used and their suffering, applying EU directive $\mathrm{N}^{\circ} 86 /$ 609, 24-11-1986.

Cortical astrocytes. Primary cultures of pure cortical rat astrocytes were prepared as previously described. ${ }^{21}$ Briefly, after removing the meninges, the cerebral cortices of post-natal Sprague-Dawley pups (P0-P2) were mechanically dissociated, placed in cell culture flasks and maintained in a humidified atmosphere incubator at $37{ }^{\circ} \mathrm{C}$ and $5 \% \mathrm{CO}_{2}$ until reaching confluence. The culture medium was replaced every 2 days. At confluence, astroglial cells were enzymatically dispersed using
trypsin-EDTA (Gibco); $2.7 \times 10^{4}$ cells per $\mathrm{mL}$ were seeded on the SF samples, SF-DC, SF-DO, SF-VD and SF-DC/MeOH, obtained from the different methods and maintained in culture medium containing $10 \%$ FBS.

Dorsal root ganglion (DRG) culture. DRG neurons from postnatal (P8-P18) rats (Sprague-Dawley) were dissected and dissociated by enzymatic digestion following procedures previously described in detail. ${ }^{22}$ An equal amount of cell suspension was dropped onto SF-VD, SF-DO, SF-DC and SF-DC/MeOH films and placed in an incubator at $37^{\circ} \mathrm{C}, 5 \% \mathrm{CO}_{2}$. Cells were maintained in DMEM (Gibco) with $10 \%$ FBS in the presence of $50 \mathrm{ng} \mathrm{mL}$ $\mathrm{NGF}$, and $1.5 \mu \mathrm{g} \mathrm{mL} \mathrm{m}^{-1}$ cytosine $\alpha$-D-arabinofuranoside (AraC, Sigma) to reduce glial cell expression.

Fluorescein diacetate assay. To reveal live cells on SF films, we used fluorescein diacetate as viability indicator. Astrocytes and DRG plated on SF-VD, SF-DO, SF-DC and SF-DC/MeOH films were incubated for $5 \mathrm{~min}$ with fluorescein diacetate solution (Sigma-Aldrich).

Imaging. Cell cultures were optically examined using a Nikon Eclipse Ti inverted microscope equipped with a $20 \times$ objective and CoolSNAP EZ CCD camera.

AlamarBlue assay. Astrocyte viability and SF sample biocompatibility were analyzed via AlamarBlue (AB) assay following the Interchim technical sheet (66941P) described previously. ${ }^{23}$ Viable cells maintain a reducing environment within their cytoplasm. AlamarBlue is a resazurin-based reagent, an oxidized form of redox indicator that is blue in color and non-fluorescent. When incubated with viable cells, the reagent is reduced and it changes color from blue to red becoming fluorescent. This change can be detected using fluorescence measurement. Cells seeded on SF-VD, SF-DO, SFDC and SF-DC/MeOH films were allowed to adhere overnight. The time course of astrocytic viability was evaluated from 1 day in vitro (DIV) to $28 \mathrm{DIV}$. On the day of the assay, $\mathrm{AB}$ reagent was added directly to culture medium at $10 \%$ volume of medium contained in each sample. After the proper incubation time at $37{ }^{\circ} \mathrm{C}, 5 \% \mathrm{CO}_{2}$, aliquots of medium/AB from each sample were transferred to a 96 multiwell plate for fluorescence measurement at $545 \mathrm{~nm}_{\mathrm{Ex}} / 590 \mathrm{~nm}_{\mathrm{Em}}$, using a Thermo Scientific Varioskan Flash Multimode Plate Reader. We included a negative control of only medium without cells to determine the background signal and a positive control of $100 \%$ reduced AlamarBlue reagent without cells. Notably, $\mathrm{AB}$ reagent does not alter healthy cells, thus whole viability time course was evaluated on the same culture of astrocytes. Data were collected from two separate experiments in quadruplicate.

Immunofluorescence. DRG cultures plated on the different samples were fixed for 15 min with $4 \%$ paraformaldehyde (PFA) in $0.1 \mathrm{M}$ phosphate-buffered saline (PBS) at room temperature (RT, 20-24 ${ }^{\circ} \mathrm{C}$ ) and then rinsed with PBS. The samples were incubated for $20 \mathrm{~min}$ in $3 \%$ bovine serum albumin (BSA) and $0.1 \%$ Triton X-100 solution, before primary antibody incubation. The immunofluorescent staining was done sequentially using primary mouse-anti-NeuN (1:100, Sigma-Aldrich) antibody, incubated overnight at RT and secondary donkey antimouse Alexa Fluor 488-conjugated (1:1000, Molecular Probes-Invitrogen) antibody, incubated for $1 \mathrm{~h}$ at RT. Coverslips 
were then mounted with Prolong Anti-Fade (Molecular ProbesInvitrogen). The fluorescent images were captured by Nikon ECLIPSE 80i microscope, equipped with appropriate fluorescence filters and a Nis-elements F300 CCD camera. Data were collected from two experiments.

Statistical analyses. Results were statistically analysed using one-way analysis of variance (ANOVA, with Bonferroni's test for mean comparisons). A statistically significant difference was reported if $p<0.05$. Data are reported as the mean \pm standard error.

\section{Conclusions}

In summary, we report the preparation of various SF films using different fabrication methods. The influence of the process parameters on the chemo-physical properties of SF films and on their interaction with neural cells was investigated. The increase in the drying temperature for SF film fabrication (SFDO film) using the standard drop casting approach (SF-DC films) showed a reduced effect on SF film stability in terms of secondary structure ( $\beta$-sheet content), wettability, mechanical resistance, dissolution and degradation profiles. However, a remarkable effect on SF film morphology and on its interaction with cortical astrocytes and DRG neurons was observed. In particular, neurite outgrowth was remarkably higher on SF-DO samples compared to the other substrates.

The microfluidic approach of vertical deposition (SF-VD film) and treatment with methanol (SF-DC/MeOH film) greatly influenced the self-assembly properties of silk protein. As a result, the $\beta$-sheet content, mechanical resistance, stability and hydrophobicity of the SF films were increased, especially for the SF-VD sample.

However, as regards the SF-cell interaction, the SF-DC/ $\mathrm{MeOH}$ sample proved to be the best candidate in terms of neural cell adhesion and astrocyte growth. The control of the chemo-physical properties of SF films plays an important role in the preparation of tailored SF-based biomaterials and composites $^{\mathbf{2 5 , 4 0}}$ suitable not only for biological and biomedical fields but also for other applications such as the recently reported silk integrating optoelectronic devices and photonics technologies. ${ }^{\mathbf{4 1 , 4 2}}$

\section{Acknowledgements}

The work was financially supported by Progetto Bandiera Fabbrica del Futuro, Silk.it, and Consorzio MIST E-R through Programma Operativo FESR 2007-2013 della Regione Emilia Romagna - Attività I.1.1, and EU through project FP7-PEOPLE2012-ITN 316832-OLIMPIA. The authors wish to thank Vincenzo Ragona for the technical support.

\section{References}

1 L. S. Nair and C. T. Laurencin, Prog. Polym. Sci., 2007, 32, 762-798.

2 O. Hakimi, D. P. Knigth, F. Vollrath and P. Vadgama, Composites, Part B, 2007, 38, 324-337.
3 B. Ratner, A. Hoffman, F. Schoen and J. Lemons, Biomaterials Science: An Introduction to Materials in Medicine, Academic Press, London, 2004.

4 B. Kundu, N. E. Kurland, S. Bano, C. Patra, F. B. Engel, V. K. Yadavalli and S. C. Kundu, Prog. Polym. Sci., 2014, 39, 251-267.

5 D. N. Rockwood, R. C. Preda, T. Yücel, X. Wang, M. L. Lovett and D. L. Kaplan, Nat. Protoc., 2011, 6, 1612-1631.

6 B. D. Lawrence, F. Omenetto, K. Chui and D. L. Kaplan, J. Mater. Sci., 2008, 43, 6967-6985.

7 T. Yucel, P. Cebe and D. L. Kaplan, Biophys. J., 2009, 97, 2044-2050.

8 G. G. Leisk, T. J. Lo, T. Yucel, Q. Lu and D. L. Kaplan, $A d v$. Mater., 2010, 22, 711-715.

9 Q. Lu, B. Zhang, M. Li, B. Zuo, D. L. Kaplan, Y. Huang and H. Zhu, Biomacromolecules, 2011, 12, 1080-1086.

10 U.-J. Kim, J. Park, C. Li, H.-J. Jin, R. Valluzzi and D. L. Kaplan, Biomacromolecules, 2004, 5, 786-792.

11 T. Yucel, M. L. Lovett and D. L. Kaplan, J. Controlled Release, 2014, 190, 381-397.

12 D.-H. Kim, Y.-S. Kim, J. Amsden, B. Panilaitis, D. L. Kaplan, F. G. Omenetto, M. R. Zakin and J. A. Rogers, Appl. Phys. Lett., 2009, 95, 133701-133703.

13 K. Inouye, M. Kurokawa, S. Nishikawa and M. Tsukada, J. Biochem. Biophys. Methods, 1998, 37, 159-164.

14 Y. Wang, H. J. Kim, G. Vunjak-Novakovic and D. L. Kaplan, Biomaterials, 2006, 27, 6064-6082.

15 M. Crespin, N. Moreau, B. Masereel, O. Feron, B. Gallez, T. Vander Borght, C. Michiels and S. Lucas, J. Mater. Sci.: Mater. Med., 2011, 22, 671-682.

16 E. Thian, Z. Ahmad, J. Huang, M. Edirisinghe, S. Jayasinghe, D. Ireland, R. Brooks, N. Rushton and S. Best, Acta Biomater., 2010, 6, 750-755.

17 G. Khang, S. J. Lee, J. H. Lee and H. B. Lee, Korea Polym. J., 1999, 7, 102-107.

18 J. H. Lee, S. J. Lee, G. Khang and H. B. Lee, J. Biomater. Sci., Polym. Ed., 1999, 10, 283-294.

19 S. J. Lee, G. Khang, Y. M. Lee and H. B. Lee, J. Colloid Interface Sci., 2003, 259, 228-235.

20 A. Leal-Egaña, G. Lang, C. Mauerer, J. Wickinghoff, M. Weber, S. Geimer and T. Scheibel, Adv. Eng. Mater., 2012, 14, B67-B75.

21 V. Benfenati, S. Toffanin, R. Capelli, L. M. Camassa, S. Ferroni, D. L. Kaplan, F. G. Omenetto, M. Muccini and R. Zamboni, Biomaterials, 2010, 31, 7883-7891.

22 V. Benfenati, K. Stahl, C. Gomis-Perez, S. Toffanin, A. Sagnella, R. Torp, D. L. Kaplan, G. Ruani, F. G. Omenetto, R. Zamboni and M. Muccini, Adv. Funct. Mater., 2012, 22, 1-14.

23 H. J. Jin, J. Park, V. Karageorgoiu, U. J. Kim, R. Valluzzi, P. Cebe and D. L. Kaplan, Adv. Funct. Mater., 2005, 15, 1241-1247.

24 K. Nagayama, Colloids Surf., A, 1996, 109, 363-374.

25 H.-J. Jin and D. L. Kaplan, Nature, 2003, 424, 1057-1061.

26 C. Dionigi, T. Posati, V. Benfenati, A. Sagnella, S. Bonetti, G. Ruani, F. Dinelli, G. Padeletti, R. Zamboni and M. Muccini, J. Mater. Chem. B, 2014, 2, 1424-1431. 
27 H. Perry, A. Gopinath, D. L. Kaplan, L. Dal Negro and F. G. Omenetto, Adv. Mater., 2008, 20, 3070-3072.

28 N. Kasoju and U. Bora, Adv. Healthcare Mater., 2012, 1, 393412.

29 X. Hu, D. L. Kaplan and P. Cebe, Macromolecules, 2006, 39, 6161-6170.

30 X. Hu, D. L. Kaplan and P. Cebe, Macromolecules, 2008, 41, 3939-3948.

31 A. S. Dimitrov and K. Nagayama, Chem. Phys. Lett., 1995, 243, 462-468.

32 K. Yamada, Y. Tsuboi and A. Itaya, Thin Solid Films, 2003, 440, 208-216.

33 T. Arai, G. Freddi, R. Innocenti and M. Tsukada, J. Appl. Polym. Sci., 2004, 91, 2383-2390.

34 A. Leal-Egana and T. Scheibel, J. Mater. Chem., 2012, 22, 14330-14336.
35 E. Servoli, D. Maniglio, A. Motta, R. Predazzer and C. Migliaresi, Macromol. Biosci., 2005, 5, 1175-1183.

36 X. Yao, R. Peng and J. Ding, Adv. Mater., 2013, 25, 5257-5286. 37 E. Vogler, J. Biomater. Sci., Polym. Ed., 1999, 10, 1015-1045.

38 Y. Arima and H. Iwata, Biomaterials, 2007, 28, 3074-3082.

39 R. P. Singh, Y. H. Cheng, P. Nelson and F. C. Zhou, Cell Transplant., 2009, 18, 55-68.

40 T. Posati, V. Benfenati, A. Sagnella, A. Pistone, M. Nocchetti, A. Donnadio, G. Ruani, R. Zamboni and M. Muccini, Biomacromolecules, 2014, 15, 158-168.

41 R. Capelli, J. J. Amsden, G. Generali, S. Toffanin, V. Benfenati, M. Muccini, D. L. Kaplan, F. G. Omenetto and R. Zamboni, Org. Electron., 2011, 12, 1146-1151.

42 M. Prosa, A. Sagnella, T. Posati, M. Tessarolo, M. Bolognesi, S. Cavallini, S. Toffanin, V. Benfenati, M. Seri, G. Ruani, M. Muccini and R. Zamboni, RSC Adv., 2014, 4, 44815-44822. 